\title{
Quantifiers in Frame Semantics
}

\author{
Laura Kallmeyer and Frank Richter
}

\begin{abstract}
We present a Lexicalized Tree Adjoining Grammar with lexical meaning specifications in Frame Semantics for the analysis of complex situations. Frame Semantics is extended by a notion of quantifier frames, which provide the basis for a translation function from frames to underspecified type-logical representations. An analysis of repetitive and restitutive readings of achievements with the adverb again demonstrates the interaction of all components of the new semantic architecture.
\end{abstract}

Keywords: frame semantics, quantifier scope, event semantics, Tree Adjoining Grammar, underspecification, syntax-semantics interface

\section{Introduction}

The goal of this paper is to develop an architecturally and conceptually clear grammar architecture that unifies lexical meaning specifications in Frame Semantics and a truth-conditional sentential semantics with generalized quantifiers and other operators favored by many formal semanticists. To obtain a framework with computational properties that are amenable to implementation, Frame Semantics and formal semantics are combined in a Tree Adjoining Grammar, and the type-logical logical representations of semantics are phrased as underspecified representations with dominance constraints.

The framework of Frame Semantics, nowadays most prominently known from the Berkeley FrameNet project [2], takes a lexicographically oriented approach to the investigation of meaning. It perceives word meanings as expressible by schematic representations of conceptual structures that stand in a web of mutual relationships, not unlike the (multiple) inheritance hierarchies of feature logical grammars. The lexical cognitive structures encode the speakers' knowledge of situations or states; moreover, they record the relationships between word senses and morphosyntactic realization patterns. With the increasing inclusion of grammatical constructions, frame semantic descriptions have decidedly moved beyond their lexical roots. Inspired by work from cognitive psychology (Barsalou, [3]), Löbner [4] takes the empirical scope of frames even further and hypothesizes that in fact the entire human cognitive system employs frames as an all-comprising single data format.

To test the viability of this research program for linguistics, a number of recent investigations have used frame semantic insights in the formulation of a syntax-semantics interface that combines the rich conceptual structures of frames with techniques of formal semantics: [5] conclude from their analysis of FrameNet that the systematicity and consistency of FrameNet's relations and 
its predictions concerning linking generalizations could benefit from frame representations whose structure reflects the internal stucture of events. They aim at a decompositional Frame Semantics that analyzes the structure of situations and events along the lines of a denotationally interpretable decompositional lexical semantics [6]. The idea of a denotational semantics for frames in terms of an intensional logic to analyze causation, situations, aktionsarten and result states is then taken up by [7] in a fragment of Lexicalized Tree Adjoining Grammar (LTAG) that captures the behavior of lexically and syntactically complex direct motion expressions, and of the dative alternation in English. The proposal combines LTAG with representations of lexical and constructional meaning, given in the form of frames that are taken to be representations of mental concepts. [7] define frames as feature structures, and the structure of their frames is suggestive about possible ways to obtain an event-logical characterization of the truth conditions of a sentence from its frame. However, the relation to truth conditions needs further clarification. Moreover, a considerable gap remains between this analysis and full-fledged Montagovian semantics: So far, frames do not foresee quantificational operators, which precludes the treatment of such standard constructs as negation or nominal phrases as generalized quantifiers.

Thus at the current stage, Frame Semantics is of limited interest to semanticists working on sentential semantics. Our main goal in this paper is to bridge this gap and connect the vision of a rich Frame Semantics embedded in a general theory of human cognition to the achievements of model-theoretic semantics. After outlining current assumptions about embedding Frame Semantics into LTAG (Section 2), we extend Barsalou frames by quantifier frames in Section 3. Intuitively, a quantifier frame embodies the idea of a concept of a quantifier, or the cognitive correlate of a quantifier. With a quantifier frame, we are able to specify frames for sentences with quantificational NPs. In order to determine the truth value of such sentences in a given model, a translation of frames into underspecified semantic representations is given, which receives its usual interpretation. The following sections demonstrate how the new architecture works: Section 4 discusses a case of a quantifier ambiguity with nested quantifiers and confirms that the syntax-semantics interface of simpler architectures without Frame Semantics can be preserved. Section 5 is dedicated to an analysis of scope ambiguities observed with repetitive and restitutive readings with the adverb again in which the lexical semantic analysis of causes and states in frames interacts in interesting ways with the quantificational properties of again. We obtain an architecture that is suitable for syntactic parsing with underspecified semantic representations with quantificational operators that benefits from lexical and constructional meaning analyses and linking in Frame Semantics.

\section{LTAG and frames semantics}

A Lexicalized Tree Adjoining Grammar (LTAG $[8,9]$ ) consists of a finite set of elementary trees. Starting from these trees, larger trees can be derived via the tree composition operations substitution (replacing a leaf with a new tree) and 

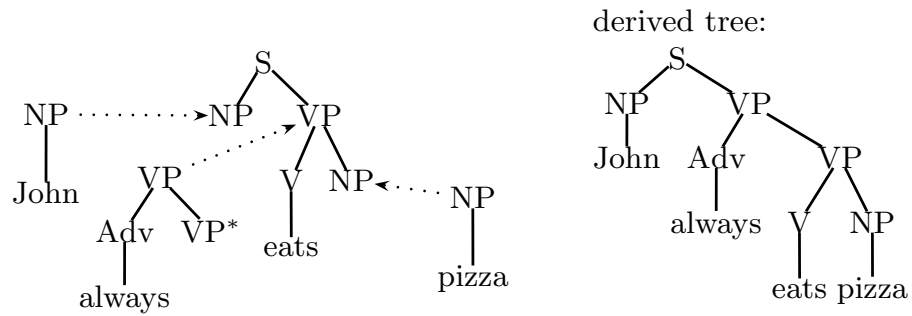

Fig. 1. A sample LTAG derivation

adjunction (replacing an internal node with a new tree). In Fig. 1 the trees for John and pizza substitute into the subject and the object slot of the tree for eats, and the tree of always adjoins to the VP node. An adjoining tree has a unique non-terminal leaf that is its foot node (marked with an asterisk). When adjoining such a tree to some node $v$, in the resulting tree, the subtree with root $v$ from the old tree ends up below the foot node.

In order to capture syntactic generalizations, the non-terminal node labels in elementary trees are usually enriched with feature structures [10]. Each node has a top and a bottom feature structure (except substitution nodes, which have only a top). Nodes in the same elementary tree can share features. Substitutions and adjunctions trigger the following unifications: In a substitution operation, the top of the root of the new tree unifies with the top of the substitution node. In an adjunction operation, the top of the root of the adjoining tree unifies with the top of the adjunction site and the bottom of the foot of the adjoining tree unifies with the bottom of the adjunction site. Furthermore, in the final derived tree, top and bottom must unify in all nodes.

Our architecture for the interface between TAG syntax and frame semantics follows ideas by [7], which in turn builds on previous approaches which link a semantic representation to an entire elementary tree and model composition by unifications triggered by substitution and adjunction [11-13]. One of the innovations of [7] is that their semantic representations are frames, expressed as typed feature structures as shown in Fig. 2. The feature I on the nodes is a syntax-semantics interface feature which stands for "individual". The assignment of semantic roles to syntactic arguments is handled by these interface features. In Fig. 2, the syntactic unifications $11 \sqcup 3$ and $2 \sqcup \sqcup 4$ identify the semantic frames of the argument NPs with the semantic roles of the verbal frame.

Following [14], the frames in [7] are formalized as multi-rooted typed feature structures with multiple base labels. In other words, some of the nodes are labelled with base labels $0,0, \ldots$, which give access to these nodes. ${ }^{1}$ Furthermore, there is no explicit type hierarchy. Instead, nodes in the frames can have several types; dependencies between types such as subtype relations and type incompatibilities are formulated in constraints in the feature logic.

${ }^{1}$ Note that when using an elementary tree with its frame in a derivation, we always use a copy with fresh base labels. 


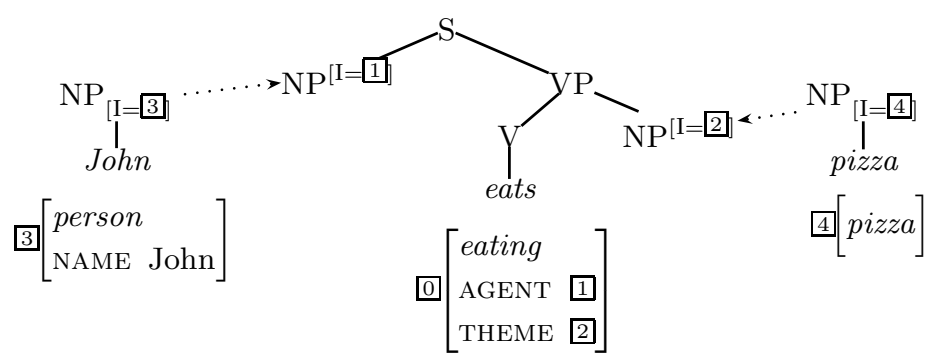

Fig. 2. Syntactic and semantic composition for John eats pizza

Concerning complexity, some restrictions on possible frame constraints are required in order to keep the system tractable. The frames that are linked to elementary trees are described within the metagrammar. Here, the possible constraints must be restricted in such a way that the existence of finite minimal models is guaranteed. To this end, we need for instance to avoid constraint loops (see also [15]). During parsing, we have to build larger frames via unifications triggered by substitution and adjunction. In order to keep this tractable, [7] assume that the constraints under consideration do not introduce new nodes to the structure. Then the complexity of unification is close to linear [14].

The extended domain of locality of LTAG, in combination with the rich factorization possibilities provided in the metagrammar through descriptions of elementary trees, permits a clean separation between lexical and constructional meaning contribution (cf. [7]).

\section{Frames for quantificational NPs}

\subsection{Quantifier frames}

Within a frame, some of the properties of a mental concept are captured by the type and others by attributes. We assume that the relation between the two arguments of binary quantifiers is captured in their frame type. This leads to the types every, most, two, etc. But what are the attributes that characterize a quantifier concept? We propose that the mental concept of a quantifier (in context) must minimally delimit the candidate concepts of its arguments, i.e. the concepts that occupy the restrictor and nuclear scope of the logical counterpart of a given quantifier concept. For this purpose, a quantifier frame contains the attribute RESTR for the maximal type of objects that the natural language quantifier in question lives on (in terms of logic: the restricting predicate), and the attributes MAXS and MINS that, in logical terms, characterize the scope window of the quantifier: The logical counterpart of the quantifier frame will scope at least over everything below the MINS value and at most over everything below the MAXS value. The embedding of the quantifier frame in a predicate frame expresses the semantic role of the syntactic constituent. 
(1)

Most dogs sleep.

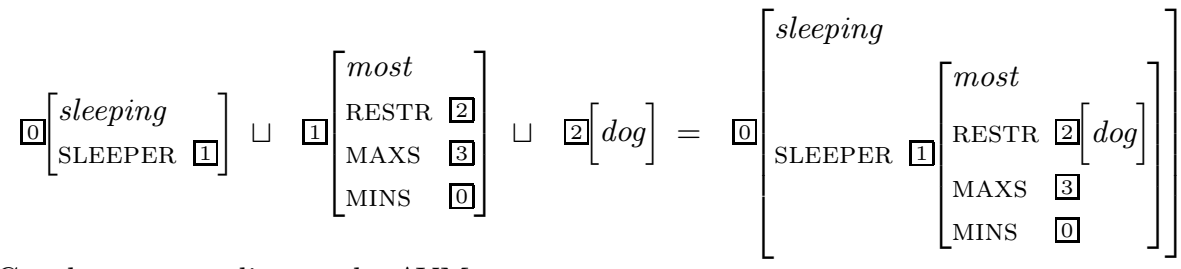

Graph corresponding to the AVM:

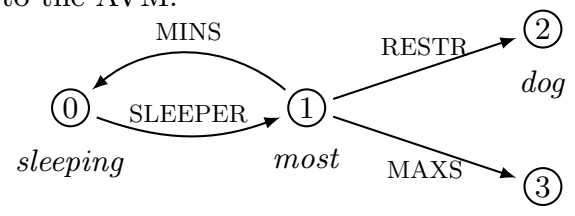

Fig. 3. Frame analysis of (1)

The cyclic structure of the frame in Fig. 3, resulting from the analysis of (1), reflects the two ways a quantifier contributes to meaning in Frame Semantics: On the one hand it (minimally) embeds some event, on the other hand it functions as an argument participant in this event and is therefore embedded in the event frame. Note that the quantifier frame does not fix the scope of the quantifier, it only records its minimal scope. In the case of (1), the minimal scope is also the actual scope. But in examples with several quantificational operators, the overall frame in effect resembles an underspecified representation of several scope orderings known from underspecified semantics.

(2) Every boy loves two girls.

In the frame in Fig. 4 of the logically ambiguous sentence (2) we also see that the semantic roles of the participants of the event are not necessarily unique: the frame labeled 1 is not only the LOVER but also the EMOTER and the EXPERIENCER, depending on how abstract a characterization of the event is adopted. This is in line with the semantic role hierarchy proposed in [16].

\subsection{Truth conditions and underspecification}

The frames in Fig. 3 and 4 do not immediately encode truth conditions that come with a model-theoretic interpretation. They are mental representations of the concepts expressed by (1) and (2). However, we can extract a predicate-logical formula with holes, labels and dominance constraints from these frames that tells us what properties the world must have in a situation where the concepts represented by the frames get instantiated. The predicate logical formulas with 


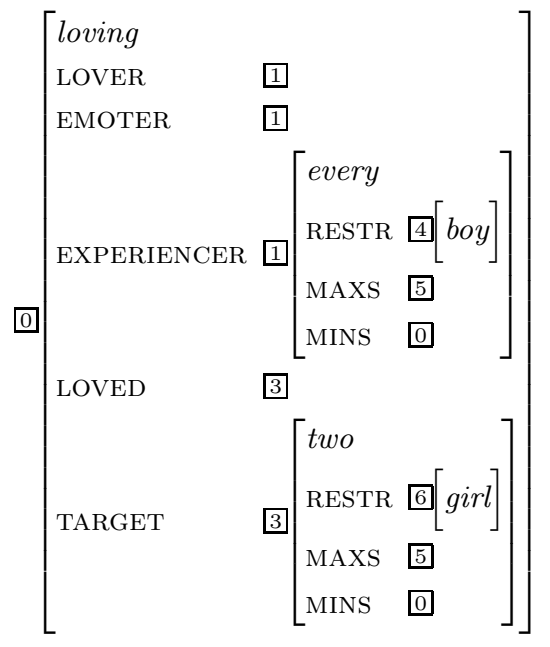

Fig. 4. Frame for (2)

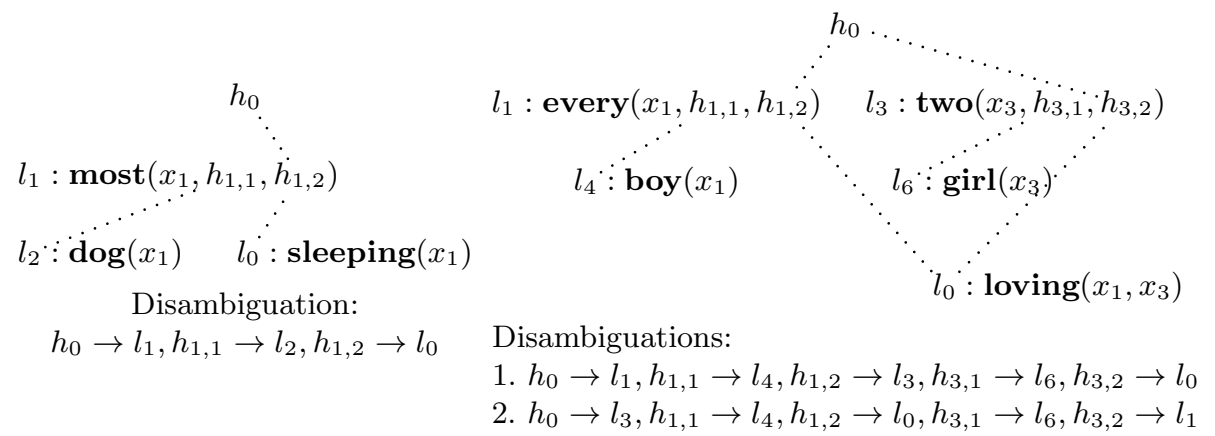

Fig. 5. Dominance constraints for (1) and (2)

holes and dominance constraints (in the sense of Hole Semantics [17]) for (1) and (2) are shown in Fig. $5 .^{2}$

Only a part of the frame is relevant for the truth conditions. For instance, the fact that the first argument of loving in Fig. 4 is characterized not only as EXPERIENCER but also via the more specific roles of EMOTER and LOVER is without counterpart in the predicate-logical formula. Furthermore, frames contain not only knowledge originating from the frames paired with elementary trees (i.e., lexical and constructional meaning) but also world knowledge. The concept of a dog in the frame in Fig. 3 comprises much more than the type $d o g$ because it comes with an entire bundle of attributes characterizing entities

\footnotetext{
${ }^{2}$ Related ideas about semantic underspecification have been framed in Underspecified Discourse Representation Theory (UDRT, [18]), the Constraint Language for Lambda Structures (CLLS, [19]) and Minimal Recursion Semantics (MRS, [20]).
} 
of type $d o g$. Since these are not relevant for our paper, they are left aside. For extracting truth conditions, only meaning contributed by elementary trees is relevant and, furthermore, only nodes with base labels or attributes relating base labeled nodes play a role.

This leaves the task to specify how to read off underspecified predicate-logical formulas for frames of type generalized-quantifier (with subtypes every, two, etc.), eventuality (with subtypes event and state) and entity (subtypes dog, boy, etc.):

- Frames of type eventuality with argument roles $\langle\arg 1\rangle,\langle\arg 2\rangle, \ldots$ (e.g., EXPERIENCER and THEME):

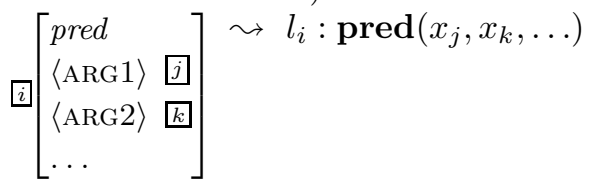

where pred is a subtype of eventuality.

- Frames of type generalized-quantifier and of type entity: ${ }^{3}$

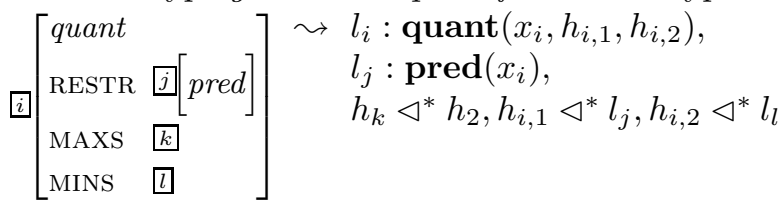

where quant is a subtype of generalized-quantifier and pred is a subtype of entity. quant and pred are the predicate logical constants corresponding to quant and pred respectively.

We assume the predicate-logical formulas to be typed. However, the types are of course not the types from our frames and there is no one-to-one correspondence between the types of frames and the type system of the predicate logic. Assuming that our variables $x_{1}, x_{2}, \ldots$ are of type $e$ in our predicate logic and holes and labels are of type $t$ (or $\langle s, t\rangle$ if we use propositions), all other types in the formulas we extract from the frames can be inferred.

Why do we pair syntactic trees with frames, generate a frame for a sentence during parsing and extract a predicate logical formula only later in a subsequent step? First of all, this architecture has advantages in terms of complexity and tractability. If we make sure that we use only feature constraints of a certain restricted type (see [7] for details), then we know that the frame unification that constitutes our semantic composition is almost linear in the size of the frames. At the same time, frame unification already acts as a filter since certain analyses will be excluded because of incompatibilities in the frames. Another important aspect of frames is that in addition to lexical and constructional meaning contributed by the building blocks of the sentence they also include world knowledge. We think it useful to be able to access this during syntactic-semantic composition and not only at some later point of interpreting a sentence in a given discourse model. Finally, following $[4,5]$ we believe that the mental concept expressed by

$\overline{3}$ The symbol $\triangleleft^{*}$ for the dominance relation is borrowed from the notation of dominance constraints in $[21,22]$. 
a sentence exists independently of the actual situation in which the sentence is supposed to hold. This mental concept is represented by the frame.

\subsection{The syntax-semantics interface}

In the previous sections, we presented the frames that we obtain from parsing and the way they (indirectly) characterize truth conditions. Concerning the syntaxsemantics interface in LTAG, the analyses from [13] can be transferred to the LTAG frame semantics architecture. Fig. 6 shows the LTAG analysis of (1). The interface feature PRED serves to pass the embedded predication frame (here of type $d o g$ ) to the restriction (feature RESTR) of the quantifier. The interface features MINS and MAXS enable passing the scope window from the verb tree to the quantifier tree. The syntax-triggered unifications are exactly the ones from Fig. 3.

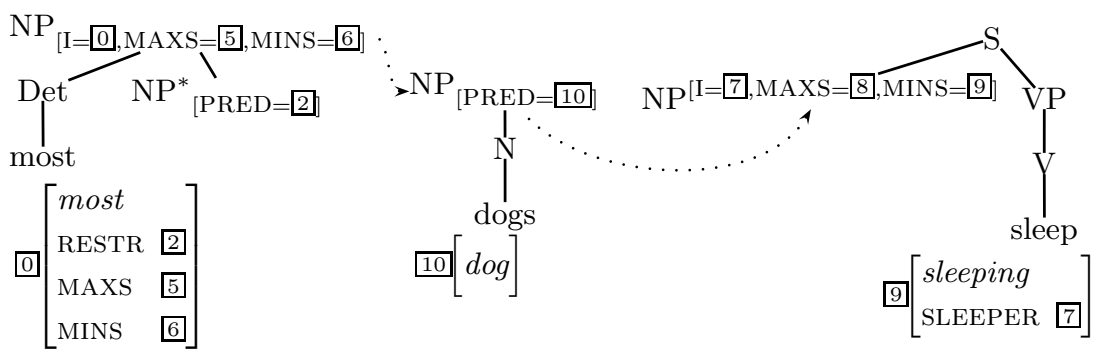

Fig. 6. Analysis for (1)

If there is more than one quantificational NP, all NPs find the same MAXS and MINS values at the substitution nodes, which entails the same scoping possibilities for all of them.

\section{Quantifiers in complex NPs}

The feasibility of the quantifier frames in our grammar architecture depends on whether they provide enough structure to derive the correct truth conditions for all and only the intended interactions between the corresponding logical operators. To ascertain that this is the case we analyze a construction (see (3)) with complex NPs with two quantifiers where the embedded quantifier can scope over the embedding one (an order sometimes called inverse linking) but a third quantifier cannot scope in between the first two (cf. [23-26]). We will show that our Frame Semantics of quantifiers is capable of reproducing the exact same readings as the ones predicted by the analysis of [13].

(3) Two policemen spy on someone from every city.

* every $>$ two $>$ some

*some $>$ two $>$ every 
The from-PP in (3) expresses the origin of a person or an object. We assume that from every city is an adjunct that adds a frame attribute ORIGIN whose value is contributed by the NP of the PP. Furthermore, it passes the label of the quantifier to which it adjoins (here 5) as the new MAXs value to any embedded NP. The MINS of the embedded NP (here 9) is the frame label to which the ORIGIN feature is added. The derivation is given in Fig. 7 and the resulting frame is shown in Fig. 8.

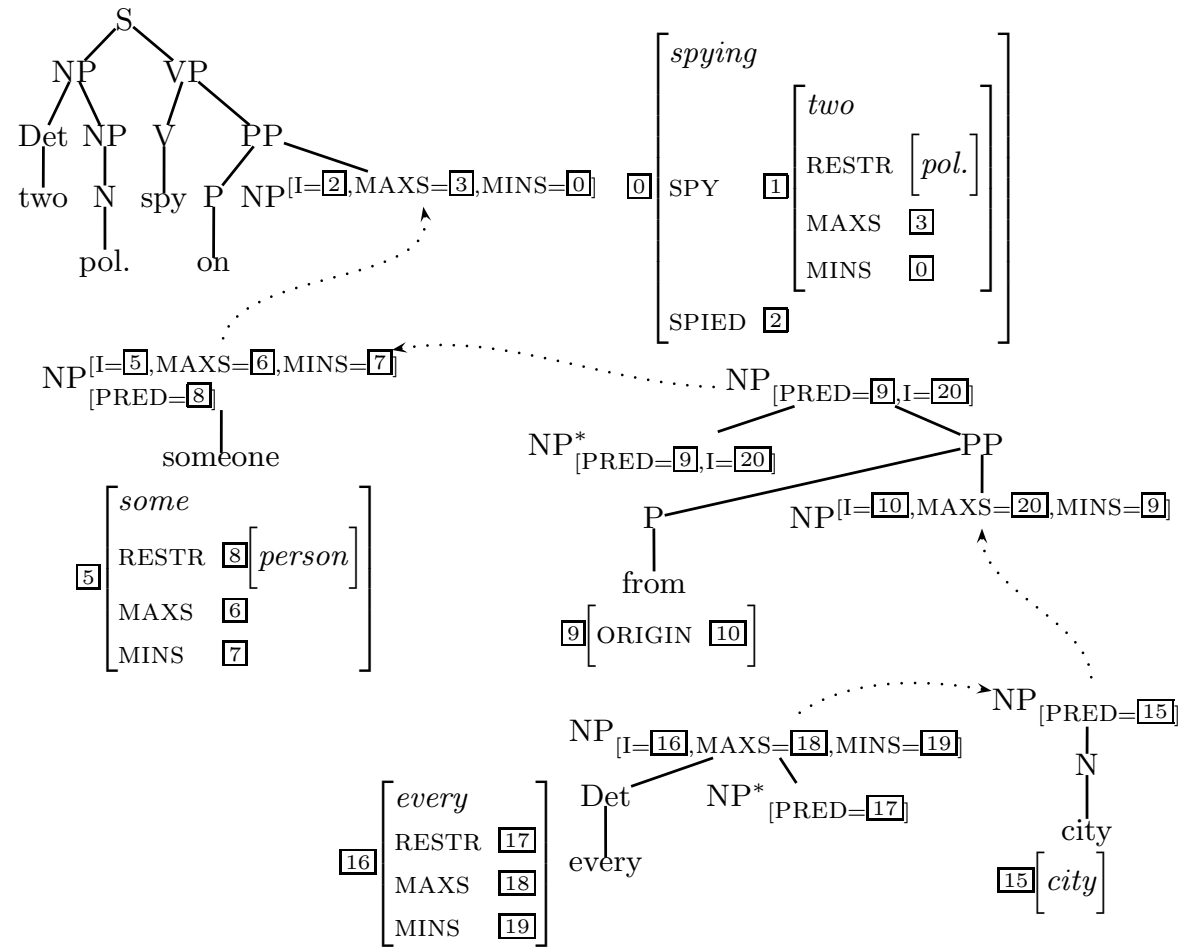

Resulting unifications (i.e., base label identifications):

$2=5=20=18$ I feature on object NP (= some frame) fills the argument of spy 0
0 $\quad$ and becomes the MAXS of the embed

3 $=6 \quad$ MAXs passing from spy to someone

$8=9=19 \quad$ PRED feature: RESTR of some becomes the MINS of from and gets passed to every

$15=17 \quad$ PRED feature: RESTR of every becomes the city frame

$10=16 \quad$ I feature: every frame fills the ORIGIN attribute

Fig. 7. Analysis for (3)

For interpretation, the frame in Fig. 8 must again be transformed into an underspecified predicate logical representation. The only addition compared to 


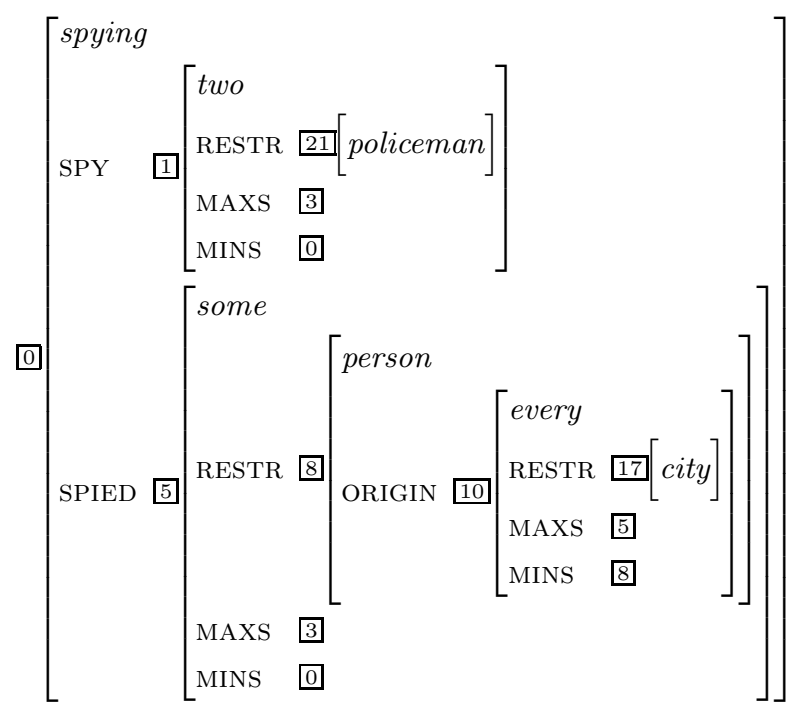

Fig. 8. Frame for (3)

the preceding section is that the attribute ORIGIN is translated to a binary predicate origin whose arguments are the two variables coming with the two nodes that it connects in the frame.

- Frames of type generalized-quantifier and of type entity:

目 $\left[\begin{array}{ll}\text { quant } & \\ \text { RESTR } & j\left[\begin{array}{ll}\text { pred } \\ \text { ATTR }\end{array}\right]\end{array}\right] \leadsto l_{j}: \operatorname{attr}\left(x_{i}, x_{k}\right)$

where quant is a subtype of generalized-quantifier and pred is a subtype of entity.

The origin-formula has the same label as the one coming from the node that the attribute modifies. In other words, we have now two expressions with the same label, $l_{8}: \operatorname{person}\left(x_{5}\right)$ and $l_{8}: \operatorname{origin}\left(x_{5}, x_{10}\right)$. They are put together in a conjunction $l_{8}: \operatorname{person}\left(x_{5}\right) \wedge \operatorname{origin}\left(x_{5}, x_{10}\right)$, in the spirit of flat semantics approaches such as Minimal Recursion Semantics (MRS, [27]). The resulting dominance constraints are depicted in Fig. 9.

The dominance constraints in Fig. 9 are resolved to exactly the desired readings. Note that it is crucial for disambiguation that the resulting structures are trees. In particular, since they are trees, nothing can be dominated by the restriction and the scope of a quantifier at the same time. In our example, if some outscopes every, then we necessarily obtain that every is dominated by the restriction $h_{5,1}$. On the other hand, if some outscopes two, then the latter necessarily is part of the scope $h_{5,2}$. Consequently, if some has wide scope, there is no scope relation between the other two quantifiers and the order some $>$ two $>$ every is correctly excluded. The other impossible reading, every $>$ two $>$ some, is excluded because the constraints state that if 


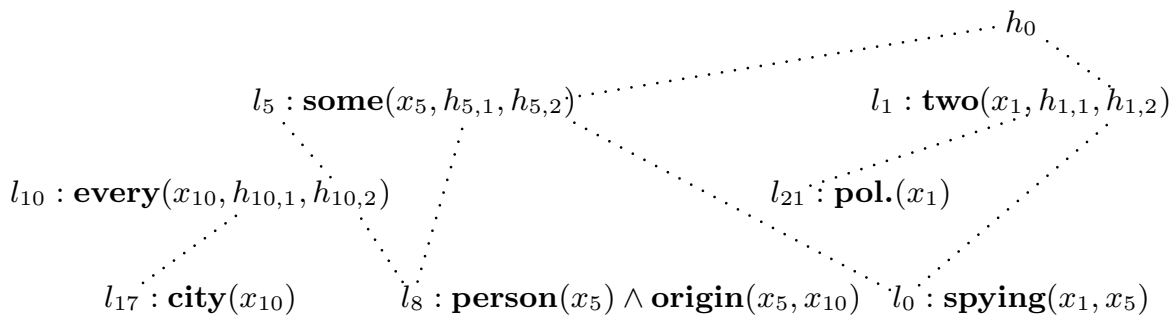

Resulting readings after disambiguation:

1. $\operatorname{some}\left(x_{5}, \operatorname{every}\left(x_{10}, \operatorname{city}\left(x_{10}\right), \operatorname{person}\left(x_{5}\right) \wedge \operatorname{origin}\left(x_{5}, x_{10}\right)\right)\right.$, two $\left(x_{1}\right.$, pol. $\left.\left.\left(x_{1}\right), \operatorname{spying}\left(x_{1}, x_{5}\right)\right)\right)$

2. $\operatorname{two}\left(x_{1}, \operatorname{pol} .\left(x_{1}\right), \operatorname{some}\left(x_{5}, \operatorname{every}\left(x_{10}, \operatorname{city}\left(x_{10}\right), \operatorname{person}\left(x_{5}\right) \wedge \operatorname{origin}\left(x_{5}, x_{10}\right)\right)\right.\right.$, $\left.\left.\operatorname{spying}\left(x_{1}, x_{5}\right)\right)\right)$

$3 . \operatorname{every}\left(x_{10}, \operatorname{city}\left(x_{10}\right), \operatorname{some}\left(x_{5}, \operatorname{person}\left(x_{5}\right) \wedge \operatorname{origin}\left(x_{5}, x_{10}\right)\right.\right.$, two $\left(x_{1}\right.$, pol. $\left.\left.\left.\left(x_{1}\right), \operatorname{spying}\left(x_{1}, x_{5}\right)\right)\right)\right)$

4. $\operatorname{two}\left(x_{1}, \operatorname{pol} .\left(x_{1}\right), \operatorname{every}\left(x_{10}, \operatorname{city}\left(x_{10}\right), \operatorname{some}\left(x_{5}, \operatorname{person}\left(x_{5}\right) \wedge \operatorname{origin}\left(x_{5}, x_{10}\right)\right.\right.\right.$, $\left.\left.\left.\operatorname{spying}\left(x_{1}, x_{5}\right)\right)\right)\right)$

Fig. 9. Dominance constraints obtained from Fig. 8 and resulting readings

every scopes over some, then its scope argument must be the some formula $\left(h_{10,2} \rightarrow l_{5}\right)$ itself, and no other quantifier may intervene.

\section{Adverbs and scope ambiguities: the case of "again"}

\subsection{Repetitive and restitutive readings}

After showing how to reconstruct operator scope ambiguities from quantifier frames, we will now turn to a phenomenon where the interaction of operator scope with the rich structure of semantic frames offers a natural basis for an interesting new analysis. When the adverb again adjoins to accomplishments like open the door or directed motion constructions like walk to the hall we observe an ambiguity between a repetitive reading (4-a) and a restitutive reading (4-b) [28].

(4) Bilbo opened the door again.

(ex. from $[28])$

a. Bilbo opened the door, and that had happened before.

b. Bilbo opened the door, and the door had been open before.

Bilbo walked to the hall again.

(ex. from [28])

a. Bilbo walked to the hall and that had happened before.

b. Bilbo walked to the hall and he had been there before.

(4) actually has a third reading that is weaker than (4-a) but stronger than (4-b), namely that Bilbo opened the door and the door had been opened before. This means it was not necessarily Bilbo who opened the door before but it is at least the second time that the state of the door changes from closed to open. 
In our event decomposition we follow $[6,29,16]$, transferring the semi-formal semantic representations used in Role and Reference Grammar (RRG) to semantic frames along the lines of $[7,5]$. (6) gives the RRG-style decompositional semantics of the verbs open and walk-to. The first is analyzed as a causation while the latter is an active accomplishment. (6-a) can be paraphrased as " $x$ performs an activity that causes $y$ to enter a state of being open". (6-b) signifies that " $x$ performs some walking activity and $x$ enters the state of being at $y$ ". In both decompositions, the coming about of the effected state and this state itself are present. This structure can be exploited to account for the restitutive readings of (4) and (5). Fig. 10 shows the translation of (6) to frames.

a. $\quad[\mathbf{d o}(x, \emptyset)]$ CAUSE [INGR open $(y)]$ b. $\quad \operatorname{do}(x, \operatorname{walk}(x)) \&$ INGR be $-\operatorname{at}(y, x)$

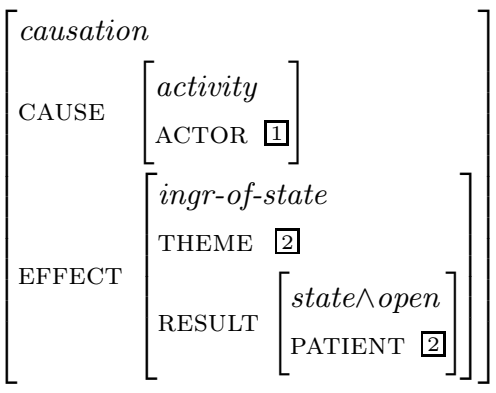

(causation) (active accomplishment)

Fig. 10. Frames for open and walk-to

According to the event decomposition in Fig. 10 the frame of open comprises three (sub-)frames for eventualities (states or events) that again can apply to, a causation frame, an ingr-of-state frame and the frame of the open state. Thus, the structure of the frame provides the basis for the observed ambiguity. In the walk-to frame, we only have two such nodes, the motion frame and the be-at frame, and there are only two readings. One of the advantages of frames is that the difference in decomposition is made explicit and the different eventualities become accessible for adverbial modification. The embedding structure explains why the restitutive reading is weaker than the intermediate reading which, in turn, is weaker than the repetitive reading.

For the semantics of again, we follow $[28,30]$ in assuming that the ambiguity between the repetitive and the restitutive reading is not due to a lexial ambiguity of again; again always indicates repetition. However, unlike these approaches we assume that the ambiguity is not a syntactic ambiguity. Instead, we derive a single syntactic tree paired with a single frame for examples such as (4) and (5). As in the quantifier scope analyses proposed in the preceding sections, the frame does not fix the ultimate scope of again. It states that the adverb minimally modifies the embedded state^open or be-at frame, respectively. Our analysis of 
(4) is shown in Fig. 11. The adverb contributes a frame of type repetition. Its minimal frame argument (the MINS value) is determined by the interface feature MINS on the VP node. This MINS value is different from the one provided for the quantifier frames at NP nodes, since the latter concern the entire event. The minimal argument of again is the resulting open state that is embedded under the effected change of state. Similarly, in the case of (5), where we do not have a causation but a walking event that is an ingr-of-state, the minimal argument provided in the construction tree is the resulting state of being at the goal of the walking. 4

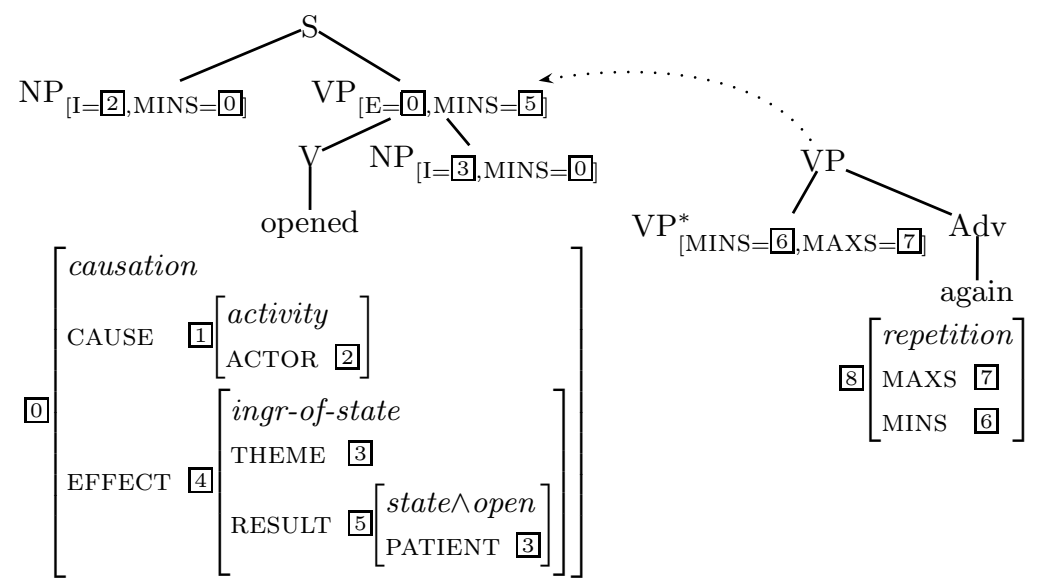

Fig. 11. Analysis of (4)

As a result of the derivation in Fig. 11, we obtain the frame and the dominance constraints in Fig. 12. Note that the frame does not have a unique root. Both the causation node and the repetition node lack incoming edges. This is different from the earlier examples where the quantifier was embedded under the event since it contributed a participant to the event.

To obtain the dominance constraints from the frames, we assume the following additional rules:

\footnotetext{
${ }^{4}$ Other authors (and an anonymous reviewer) note that only few adverbs seem to have access to sublexical modification of result states (for an overview and discussion, see [28]). For example, (i) cannot mean that Bilbo opened the door once and then it always stayed open.
}

(i) Bilbo always opened the door.

Appropriate restrictions can be stated in our framework, but we do not want to commit to a particular formulation here. For other relevant examples of sublexical modification such as the ambiguous noun phrase a beautiful dancer (a dancer who dances beautifully vs. a beautiful-looking dancer), see [31]. 
Frame:

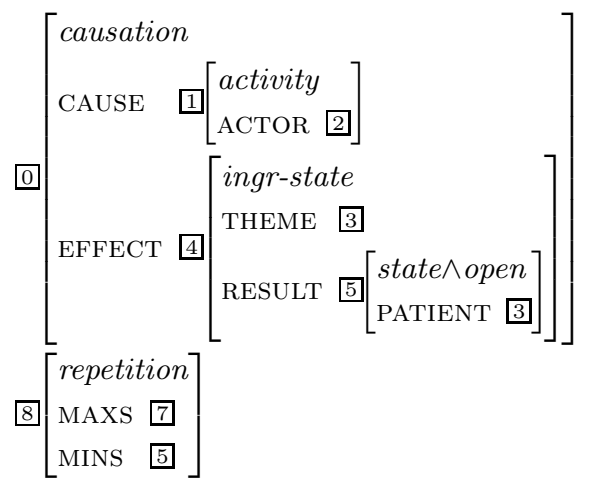

Dominance constraints:

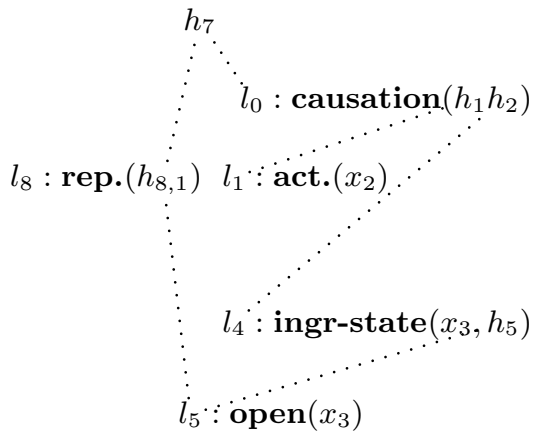

Disambiguations (minimal models of the dominance constraints):

1. repetition(causation(activity $\left.\left(x_{2}\right), \operatorname{ingr-state}\left(x_{3}, \operatorname{open}\left(x_{3}\right)\right)\right)$ )

2. causation(activity $\left(x_{2}\right), \operatorname{repetition}\left(\operatorname{ingr-state}\left(x_{3}, \operatorname{open}\left(x_{3}\right)\right)\right)$ )

3 . causation(activity $\left(x_{2}\right)$, ingr-state $\left(x_{3}\right.$, repetition $\left.\left(\operatorname{open}\left(x_{3}\right)\right)\right)$

Fig. 12. Frame and dominance constraints for (4)

- Frames of type event-quantification:

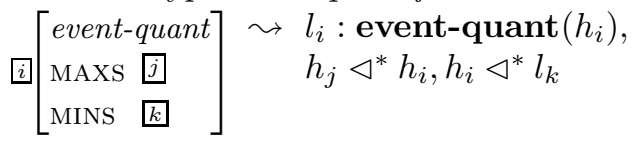

where event-quant is a subtype of event-quantification (for instance repetition.

- Frames of type eventuality with entity-valued argument roles $\langle\arg 1\rangle,\langle\arg 2\rangle$, ... (e.g., EXPERIENCER and THEME) and eventuality-valued argument roles $\langle$ event 1$\rangle,\langle$ event 2$\rangle, \ldots$ (e.g., CAUSE and EFFECT):

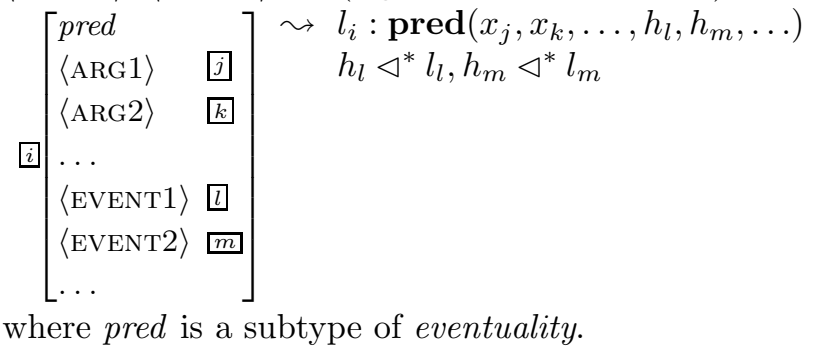

\subsection{Interaction with quantificational NPs}

By adding a quantifier to $(4) /(5)$, we can provoke an additional semantic ambiguity due to an interaction with the adverb again as demonstrated in (7) and (8), with the new NP quantifier in object position and in subject position, respectively. In both cases, a reading where again outscopes the quantifier is possible: In (7), this reading signifies that it happened again that Bilbo opened more than half of the doors, but he did not necessarily open the same as before. In (8), wide 
scope of again signifies that it was again the case that there were two dwarfs (not necessarily the same as before) that walked to the hall.

(7) Bilbo opened more than half of the doors again.

(8) Two dwarfs walked to the hall again.

Let us consider the analysis of (8). The adjoining again picks the label of the result state as its minimal argument frame while the minimal argument of the quantifier two dwarfs is the label of the entire walking frame. As basis for this, the elementary construction of a directed motion with a goal PP provides the two different labels as MINS values at the VP node and the NP/PP nodes, respectively. We obtain the frame and the dominance constraints in Fig. $13 .^{5}$

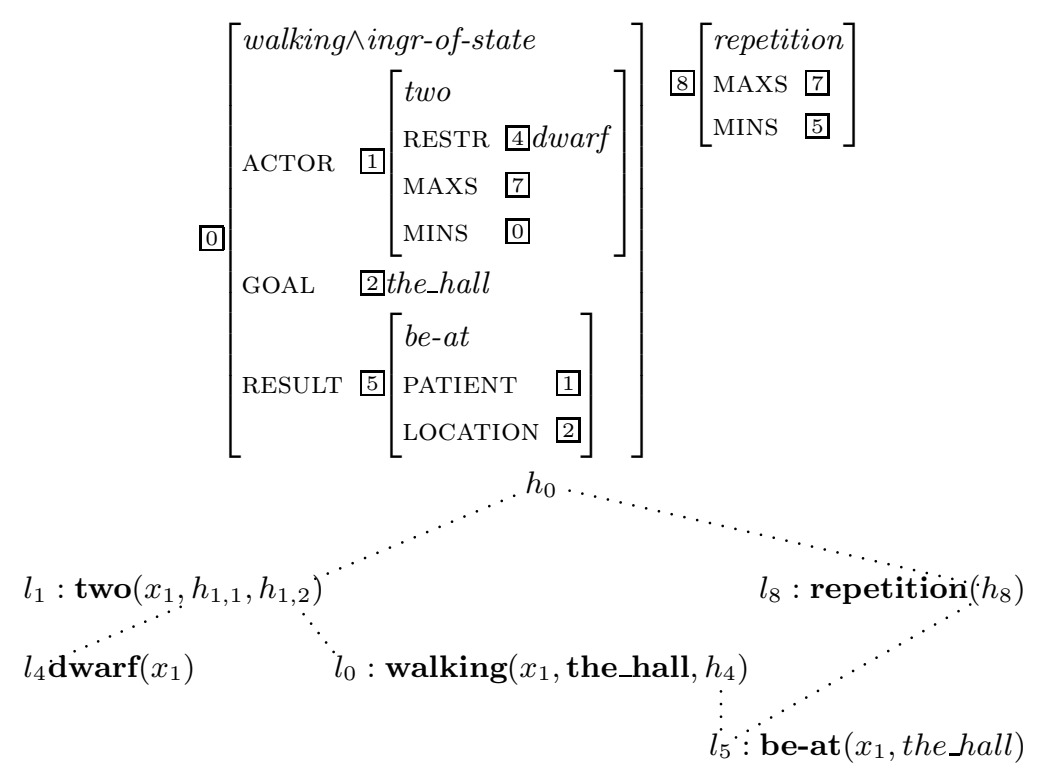

Fig. 13. Frame for (8)

The analysis of again proposed in this section has demonstrated that a combination of the detailed event decomposition of frames and the techniques of dominance constraints in underspecified scope representations paves the way to an elegant account of challenging scope phenomena. Our framework provides an analysis that avoids either lexical ambiguity or structural ambiguity, one of which was necessary in the two earlier types of accounts $[32,30,28]$.

\footnotetext{
${ }^{5}$ The analysis of the definite article is left aside in this paper.
} 


\section{Conclusion}

We added quantifier frames to Frame Semantics and defined a translation from frames to underspecified semantic representations that lead to resolved representations with a conventional interpretation in models. With its integration in LTAG a new architecture emerged in which a Frame Semantics with fine-grained lexical decompositions of situations as frames supports a well-defined logical semantics with quantificational and intensional operators.

We distinguished a level of representation with frames as feature structures from a derived level of interpreted logical representations. Frames are taken to be cognitive representations of concepts, world knowledge and situational knowledge. When frame semantics represents a dog, it aims at the concept of a dog and an agent's knowledge of dogs including the range of their size or that they are carnivores etc. Type logical semantics on the other hand provides a predicate denoting the set of dogs in a model. Separating frames and logical semantics is therefore crucial to do justice to the fundamentally different nature of the two. As lexical representations, frames are supposed to reflect how speakers decompose and represent the meaning of lexical units. The syntax-semantics interface connects syntactic units to frames, which are in turn systematically related to the interpreted logical representations. In the grammatical system, frames are prominently responsible for the assignment of semantic roles. We showed that their structure is sufficiently rich to define a translation function to underspecified logical representations that explicate the possible scope relations between the quantifiers associated with the fillers of semantic roles or adverbial modifiers.

In future research it would be interesting to investigate in more detail how the comprehensive knowledge resources that cognitive scientists assume for frames are related to linguistic representations, and what their role is in reasoning.

\section{References}

1. Gamerschlag, T., Gerland, D., Osswald, R., Petersen, W., eds.: Frames and Concept Types. Volume 94 of Studies in Linguistics and Philosophy. Springer (2014)

2. Fillmore, C.J., Johnson, C.R., Petruck, M.R.: Background to FrameNet. International Journal of Lexicography 16(3) (2003) 235-250

3. Barsalou, L.W.: Frames, concepts, and conceptual fields. In Lehrer, A., Kittay, E.F., eds.: Frames, Fields, and Contrasts. New Essays in Semantic and Lexical Organization. Lawrence Erlbaum Associates, Hillsdale, New Jersey (1992) 21-74

4. Löbner, S.: Evidence for frames from human language. [1] 23-67

5. Osswald, R., Van Valin, R.D.: FrameNet, frame structure, and the syntaxsemantics interface. [1] 125-156

6. Dowty, D.R.: Word Meaning and Montague Grammar. D. Reidel Publishing Company (1979)

7. Kallmeyer, L., Osswald, R.: Syntax-driven semantic frame composition in Lexicalized Tree Adjoining Grammars. Journal of Language Modelling 1(2) (2013) $267-330$ 
8. Joshi, A.K., Schabes, Y.: Tree-Adjoning Grammars. In Rozenberg, G., Salomaa, A., eds.: Handbook of Formal Languages. Springer, Berlin (1997) 69-123

9. Abeillé, A., Rambow, O.: Tree Adjoining Grammar: An Overview. In Abeillé, A., Rambow, O., eds.: Tree Adjoining Grammars: Formalisms, Linguistic Analysis and Processing. CSLI (2000) 1-68

10. Vijay-Shanker, K., Joshi, A.K.: Feature structures based tree adjoining grammar. In: Proceedings of COLING, Budapest (1988) 714-719

11. Gardent, C., Kallmeyer, L.: Semantic Construction in FTAG. In: Proceedings of EACL 2003, Budapest (2003) 123-130

12. Kallmeyer, L., Joshi, A.K.: Factoring Predicate Argument and Scope Semantics: Underspecified Semantics with LTAG. Research on Language and Computation 1(1-2) (2003) 3-58

13. Kallmeyer, L., Romero, M.: Scope and situation binding in LTAG using semantic unification. Research on Language and Computation 6(1) (2008) 3-52

14. Hegner, S.J.: Properties of Horn clauses in feature-structure logic. In Rupp, C.J., Rosner, M.A., Johnson, R.L., eds.: Constraints, Language and Computation. Academic Press, San Diego, CA (1994) 111-147

15. Carpenter, B.: The Logic of Typed Feature Structures. Cambridge University Press (1992)

16. Van Valin, Jr., R.D.: Exploring the Syntax-Semantics Interface. Cambridge University Press (2005)

17. Bos, J.: Predicate logic unplugged. In Dekker, P., Stokhof, M., eds.: Proceedings of the 10th Amsterdam Colloquium. (1995) 133-142

18. Reyle, U.: Dealing with ambiguities by underspecification: Construction, representation and deduction. Journal of Semantics 10 (1993) 123-179

19. Egg, M., Koller, A., Niehren, J.: The Constraint Language for Lambda Structures. Journal of Logic, Language and Information 10(4) (2001) 457-485

20. Copestake, A., Flickinger, D., Pollard, C., Sag, I.A.: Minimal Recursion Semantics: An introduction. Research on Language and Computation 3 (2005) 281-332

21. Althaus, E., Duchier, D., Koller, A., Mehlhorn, K., Niehren, J., Thiel, S.: An efficient graph algorithm for dominance constraints. Journal of Algorithms 48(1) (2003) 194-219

22. Koller, A., Niehren, J., Treinen, R.: Dominance Constraints: Algorithms and Complexity. In: Proceedings of the Third International Conference on Logical Aspects of Computational Linguistics (LACL), Grenoble, France (December 1998)

23. Heim, I., Kratzer, A.: Semantics in Generative Grammar. Blackwell (1998)

24. Barker, C.: Continuations and the Nature of Quantification. Natural Language Semantics 10 (2002) 167-210

25. Sauerland, U.: DP is not a scope island. Linguistic Inquiry 36 (2004) 303-314

26. Joshi, A.K., Kallmeyer, L., Romero, M.: Flexible Composition in LTAG: Quantifier Scope and Inverse Linking. In Muskens, R., Bunt, H., eds.: Computing Meaning Volume 3. Volume 83 of Studies in Linguistics and Philosophy. Springer (2007) 233-256

27. Copestake, A., Flickinger, D., Pollard, C., Sag, I.A.: Minimal Recursion Semantics: An introduction. Research on Language and Computation 3 (2005) 281-332

28. Beck, S.: There and back again: A semantic analysis. Journal of Semantics 22 (2005) 3-51

29. Van Valin, Jr., R.D., LaPolla, R.: Syntax: Structure, meaning and function. Cambridge University Press (1997)

30. von Stechow, A.: The different readings of wieder "again": A structural account. Journal of Semantics 13 (1996) 87-38 
31. Egg, M.: Mismatches at the syntax-semantics interface. In Müller, S., ed.: Proceedings of the 11th International Conference on Head-Driven Phrase Structure Grammar, Center for Computational Linguistics, Katholieke Universiteit Leuven, Stanford, CA, CSLI Publications (2004) 119-139

32. Fabricius-Hansen, C.: Wieder ein wieder? Zur Semantik von wieder. In Bäuerle, R., Schwarze, C., von Stechow, A., eds.: Meaning, Use, and Interpretation of Language. Walter de Gruyter (1983) 97-120 